\title{
DR-17
}

\section{MOLECULAR COMPLEX OF QUERCETIN WITH HEDERASAPONIN C}

\author{
L. A. Yakovishin, ${ }^{1}$ V. D. Ratnikov, ${ }^{1}$ P. I. Bazhan, ${ }^{1}$ V. I. Grishkovets, ${ }^{2}$ E. N. Korzh ${ }^{1}$ \\ ${ }^{1}$ Sevastopol State University, University St. 33, Sevastopol, 299053, Russia; \\ ${ }^{2}$ V.I. Vernadsky Crimean Federal University, Vernadsky Ave. 4, Simferopol, 295007, Russia. \\ E-mail: chemsevntu@ rambler.ru
}

\begin{abstract}
Quercetin (Quer) is one of the most famous flavonols ${ }^{1}$. Quer was founded in different plants. Quer has P-vitamin activity and exhibits antioxidant, anti-inflammatory, antispasmodic, antisclerotic, diuretic, and antitumor effects. ${ }^{1,2}$

Previously, we have studied the molecular complex of Quer with triterpene glycoside glycyram (monoammonium salt of glycyrrhizic acid) $^{3}$. Triterpene glycosides from licorice and ivy are one of the most affordable saponins. However, molecular complexes of Quer with ivy triterpene glycosides are not described. Triterpene glycoside hederasaponin $\mathrm{C}$ (hederacoside $\mathrm{C}$, hederagenin 3-O- $\alpha$-Lrhamnopyranosyl-( $1 \rightarrow 2)$-O- $\alpha$-L-arabinopyranosyl-28-O- $\alpha$-L-rhamnopyranosyl-( $1 \rightarrow 4)-\mathrm{O}-\beta$-D-

glucopyranosyl-( $1 \rightarrow 6)-\mathrm{O}-\beta$-D-glucopyranoside, HedC) was discovered in the most species of the ivy genus Hedera L. (Araliaceae), in which it is the dominant saponin.

The Quer-HedC complex composition was determined by the method of isomolar series at 256 and $370 \mathrm{~nm}$. This method gave a molar ratio $\approx 2.0$, which corresponded to a $1: 2$ complex of Quer with $\mathrm{HedC}$, respectively (in 2: 8 mixture of $96 \% \mathrm{EtOH}$ and aqueous phosphate buffer with $\mathrm{pH} 7.2(\mathrm{v} / \mathrm{v})$ ).

The molecular complexation of Quer with HedC was studied by ATR FT-IR spectroscopy. It was shown that hydrogen bonds are formed between $\mathrm{OH}$ groups of complex components, and by $\mathrm{C}=\mathrm{O}$ group of Quer and carbohydrate $\mathrm{OH}$ groups of HedC: $(\mathrm{H}) \mathrm{O} \cdots \mathrm{H}-\mathrm{O}$ and $\mathrm{C}=\mathrm{O}_{\text {Quer }} \cdots \mathrm{H}-\mathrm{O}$ HedC.
\end{abstract}
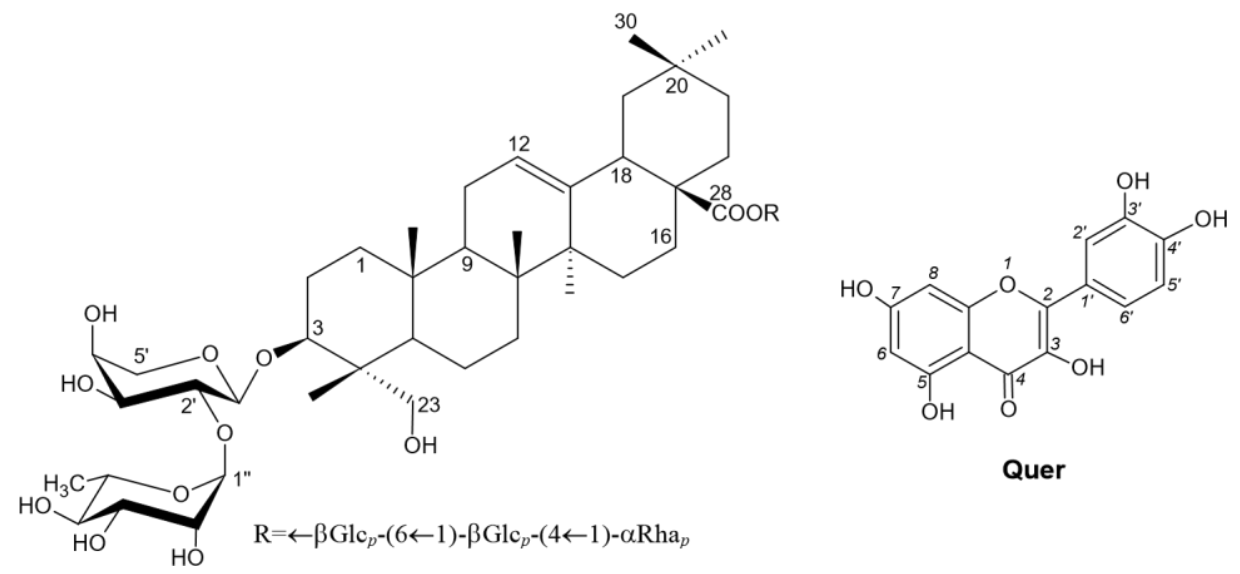

HedC

\section{References}

1. Boots A. W. Health effects of quercetin: from antioxidant to nutraceutical / A. W. Boots, G. R. M. M. Haenen, A. Bast // European Journal of Pharmacology. - 2008. - Vol. 585, Iss. 2-3. - P. 325-337.

2. Dietary wine phenolics catechin, quercetin, and resveratrol efficiently protect hypercholesterolemic hamsters against aortic fatty streak accumulation / C. Auger, P. L. Teissedre, P. Gérain [et al.] // Journal of Agricultural and Food Chemistry. 2005. - Vol. 53, Iss. 6. - P. 2015-2021.

3. Yakovishin L. A. Molecular complex of quercetin with glycyram / L. A. Yakovishin, E. N. Korzh // AIP Conference Proceedings. - 2019. - Vol. 2063. - 040066. 